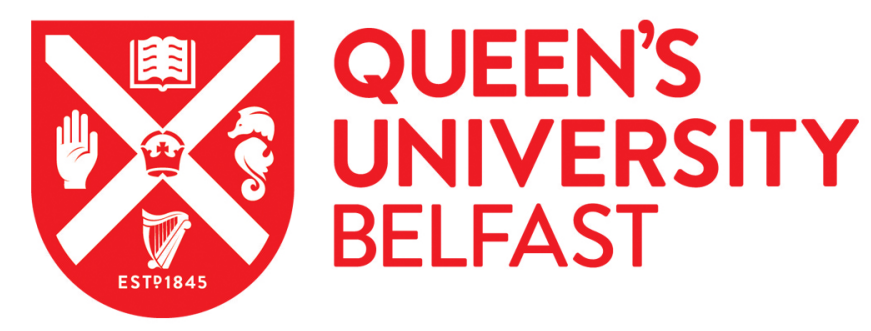

\title{
Efficient and Effective Multi-Modal Queries through Heterogeneous Network Embedding
}

Duong, C. T., Nguyen, T. T., Yin, H., Weidlich, M., Mai, T. S., Aberer, K., \& Nguyen, Q. V. H. (2021). Efficient and Effective Multi-Modal Queries through Heterogeneous Network Embedding. IEEE Transactions on Knowledge and Data Engineering. https://doi.org/10.1109/TKDE.2021.3052871

Published in:

IEEE Transactions on Knowledge and Data Engineering

Document Version:

Peer reviewed version

Queen's University Belfast - Research Portal:

Link to publication record in Queen's University Belfast Research Portal

Publisher rights

Copyright 2021 IEEE. This work is made available online in accordance with the publisher's policies. Please refer to any applicable terms of use of the publisher.

\section{General rights}

Copyright for the publications made accessible via the Queen's University Belfast Research Portal is retained by the author(s) and / or other copyright owners and it is a condition of accessing these publications that users recognise and abide by the legal requirements associated with these rights.

Take down policy

The Research Portal is Queen's institutional repository that provides access to Queen's research output. Every effort has been made to ensure that content in the Research Portal does not infringe any person's rights, or applicable UK laws. If you discover content in the Research Portal that you believe breaches copyright or violates any law, please contact openaccess@qub.ac.uk. 


\title{
Efficient and Effective Multi-Modal Queries through Heterogeneous Network Embedding
}

\author{
Chi Thang Duong ${ }^{1}$, Thanh Tam Nguyen ${ }^{1}$, Hongzhi Yin², Matthias Weidlich², \\ Thai Son Mai ${ }^{4}$, Karl Aberer ${ }^{1}$, Quoc Viet Hung Nguyen ${ }^{5}$ \\ ${ }^{1}$ Ecole Polytechnique Federale de Lausanne, ${ }^{2}$ The University of Queensland, ${ }^{3}$ Humboldt-Universität zu Berlin, \\ ${ }^{4}$ Queen's University Belfast, ${ }^{5}$ Griffith University
}

\begin{abstract}
The heterogeneity of today's Web sources requires information retrieval (IR) systems to handle multi-modal queries. Such queries define a user's information needs by different data modalities, such as keywords, hashtags, user profiles, and other media. Recent IR systems answer such a multi-modal query by considering it as a set of separate uni-modal queries. However, depending on the chosen operationalisation, such an approach is inefficient or ineffective. It either requires multiple passes over the data or leads to inaccuracies since the relations between data modalities are neglected in the relevance assessment. To mitigate these challenges, we present an IR system that has been designed to answer genuine multi-modal queries. It relies on a heterogeneous network embedding, so that features from diverse modalities can be incorporated when representing both, a query and the data over which it shall be evaluated. By embedding a query and the data in the same vector space, the relations across modalities are made explicit and exploited for more accurate query evaluation. At the same time, multi-modal queries are answered with a single pass over the data. An experimental evaluation using diverse real-world and synthetic datasets illustrates that our approach returns twice the amount of relevant information compared to baseline techniques, while scaling to large multi-modal databases.
\end{abstract}

Index Terms—query embedding, graph embedding, heterogeneous information network

\section{INTRODUCTION}

The Web is built from heterogeneous representations of information, including texts, images, and videos. Recently, the rise of social media led to further rich and complex data modalities gaining increasing importance, among them user profiles, hashtags, and keywords. Given the rich semantics of heterogeneous data and the large data volumes faced in practice, retrieval of relevant information remains a challenging task for data on the Web [1].

A multi-modal information retrieval (IR) system takes as input a set of multi-modal queries and returns a ranked list of elements selected from a corpus of multi-modal data. It generalizes the model for uni-modal queries over textual corpora of webpages and documents, which has been the primary concern of IR systems for several decades [2]. Various systems that support non-textual retrieval have been proposed recently [2], [3]. Here, a user searches for information represented as images or audio based on queries that include several modalities beyond textual keywords.

Existing IR systems deal with heterogeneity of the queried data by constructing a vectorized representation of it. However, these systems lack support for multi-modal queries and focus on textual queries. This has negative implications on the retrieval accuracy due to the inherent textual ambiguity. In addition, textual queries limit users in effectively formulating their information needs [4]. Consider a scenario in which a user searches for a song in a music library. Instead of relying only on keywords without any semantics, a multi-modal query supports the explicit specification of a composer or album of the song. By incorporating these modalities, retrieval becomes more effective.

Multi-modal queries as outlined above are different from faceted search. The latter classifies data into semantic categories (facets) to filter results after retrieval [5]. Multi-modal queries, in turn, incorporate modalities in the identification of the relevant results that shall be retrieved.

Recently, first IR systems started to support the specification of multi-modal queries [3], [6]. Yet, they do not genuinely evaluate multi-modal queries, but consider them as a combination of several uni-modal queries, each of which covering a different modality. Then, the retrieval results are constructed by combining the answers to different unimodal queries via fusion. To this end, late fusion [7], [8], [9] combines the results obtained for each modality in isolation, which is inefficient as it requires several passes over the data. Another approach is using early fusion [10], [11], [12], which embeds uni-modal queries based on different feature vectors that are combined with an aggregation function. While such an approach requires only a single pass over the data, it ignores the relations between modalities. Hence, it breaks the semantic structure of the data, so that features of retrieved elements may not belong to the same data entity.

In this paper, we provide a novel angle for multi-modal IR based on a vectorized representation of a heterogeneous information network (HIN). The HIN model captures the rich semantics of both, multi-modal data and queries. Our contributions are summarized as follows.

- A model for heterogeneous data: We introduce a representation of heterogeneous data based on HINs to capture the semantic relations between data of different modalities of the same entity [13], [14]. This includes a 
network embedding model to produce a feature vector for each node in the HIN based on its modality.

- Multi-modal query model: We propose a query model that enables users to incorporate different modalities. We construct a query embedding based on the HIN embedding of the queried data through novel mapping and combination operators. This way, we ensure that query and data embeddings are defined over the same space, which facilitates an accurate relevance assessment.

- Scalable information retrieval: Striving for query evaluation over large-scale datasets, we propose a parallel variant of our network embedding. It divides a HIN into subnetworks and performs embedding on each of them separately. However, since the resulting embeddings may belong to different spaces, we also show how to construct a unified embedding space.

We evaluated the proposed approach with a set of diverse real-world and synthetic datasets. Our techniques turn out to be both efficient, scaling linearly to hundreds of thousands of data elements, and effective, retrieving twice the amount of relevant tuples compared to baseline techniques.

The remainder of the paper is organized as follows. Next, we motivate and formulate the addressed problem in $\S 2$. Then, $\S 3$ gives an overview of our approach. The embedding of HINs is discussed in $\S 4$, while query embedding is explained in $\S 5$. $\S 6$ describes the scalable version of our approach. Experimental results are presented in $\$ 7 . \oint 8$ reviews related work, before we conclude in $\S 9$.

\section{Problem formulation}

Below, we first present a motivating example (\$2.1) and a formal model ( $\$ 2.2)$, before we formulate the problem addressed in this work (§2.3).

\subsection{Motivation}

Running example. Let us consider the following setting, in which a user searches for a song.

Example 1. A user searches for the song 'Jenny of Oldstones', but does not remember its title. The user recalls that it is a song from the soundtrack of 'Game of Thrones'. Yet, using this as a keyword is not effective, since the retrieved results are mostly related to the movie itself. Specifying a query that explicitly includes a modality 'music album' with the value being 'Game of Thrones' is more precise, but still leads to many songs that have been included in the soundtrack. A user may then try to identify the song by querying for other modalities. For instance, the user may recall some terms of the lyrics, such as 'winter'. Also, the user may search for songs of specific singers, e.g., 'Florence', or composers, such as 'Dan Weiss'. Yet, considering each of these queries in isolation, long lists of songs will be returned, as each modality on its own does not enable a precise identification of a specific song.

The above is a common example of a retrieval task in which a user needs to specify multiple queries in several modalities. In the end, a user has to identify the relevant results in large collections of retrieved elements, with the risk of not finding the requested element at all. Note that we use a music database as an example for multi-modal information retrieval. Yet, our techniques are independent of a specific domain since they adopt a generic graph-based representation of heterogeneous data.

Limitations of traditional approaches. Traditional IR systems fail to satisfy information requirements such as illustrated above, as they support search over one modality only. Consequently, they potentially miss out on important semantics. The limitation is not bound to modalities that are textual, but is also observed for other types of data, such as videos and images. Again, limiting a user to formulate a single, unimodal query may make it impossible to capture the users' true intention. In practice, this limitation is often addressed by incremental algorithms, such as query refinement and user feedback [15]. Since the user intent cannot be specific precisely, multiple passes over the data are needed to refine a search result.

Against this background, it was suggested to combine the results of several uni-modal queries [16]. Yet, fusing search results of different queries neglects that the relevance ranking obtained for different modalities might be incompatible. In addition, such an approach does not scale well to large data, since it requires multiple passes over the data to answer the uni-modal queries. A different angle is followed by IR systems with 'querying by example' functionality over multimedia databases. Here, a user may conduct retrieval by specifying a data sample [17]. Yet, the respective systems are highly domain-specific and are not applicable in the absence of such a sample, as in Example 1.

\subsection{A Multi-Modal Query Model}

We design a model for multi-modal queries as follows.

Data model. We capture heterogeneous data by a set of tuples $D=\left\{d_{1}, \ldots, d_{n}\right\}$ and a set of attributes $\mathcal{A}=\left\{A_{1}, \ldots, A_{m}\right\}$. Each attribute is considered as a modality, which defines a set of possible attribute values $A_{i}=\left\{t_{i 1}, t_{i 2}, \ldots\right\}$. Each tuple is defined as a vector $d=\left(a_{1}, \ldots, a_{m}\right)$ where $a_{i}$ is a subset of $A_{i}$. This model is generic and applicable for various types of data [18]. For instance, a set of relational tables can be flatten into a single table and domain-specific features, such as the audio embedding of a song, may also be represented through a concatenation of features in the tuple vector [19].

Example 2. A song is be modelled as a tuple of (title, album, lyrics, singer, composer). The song described in Example 1 is then represented as (\{Jenny of Oldstones $\},\{$ Game of Thrones $\}$, $\{$ winter, etc. $\},\{$ Florence $\},\{$ Dan Weiss, etc. $\})$.

Query model. A user's query over multi-modal data is captured by a set of uni-modal queries $\mathcal{Q}=\left\{Q_{1}, \ldots, Q_{m}\right\}$. Each of them relates to a different modality and specifies a subset of its domain, $Q_{i} \subseteq A_{i}$. With all queries except one being empty, the model captures the setting of traditional uni-modal information retrieval. Multi-modal information retrieval happens, if $\mathcal{Q}$ contains at least two non-empty queries. We denote the set of all values of all modalities as $\Omega=\cup_{A \in \mathcal{A}} A$. Similarly, the set of all queried values is $q=\cup_{Q \in \mathcal{Q}} Q$, which is a subset of $\Omega$.

Note that, in addition to user-specified queries, this model also supports the paradigm of 'querying by example'. In that case, the queried values are derived directly from a selected sample of the database. 
album=GoT, lyrics=\{winter,$\quad J=-\mathbf{M}$ m - , etc.

Fig. 1. An example of a multi-modal query.

Example 3. The user's information need in Example 1 can be captured by a multi-modal query that comprises several uni-modal queries: $Q_{\text {lyrics }}=\{$ winter $\}, Q_{\text {album }}=\{$ Game of Thrones $\}$, $Q_{\text {singer }}=\{$ Florence $\}$, and $Q_{\text {composer }}=\{$ Dan Weiss $\}$. $A$ user could even provide an audio sample, so that query $Q_{\text {audio }}=\{w a v$-file $\}$ would point to an audio file, if available. Fig. 1 illustrates an example of a multi-modal query.

\subsection{Problem Statement}

Given the above model, we consider the problem of identifying tuples that are most relevant for a multi-modal query. Formally, we capture the underlying notion of relevance by a function $f^{*}: \mathcal{D} \times \Omega \rightarrow \mathbb{R}$ that assigns a relevance score to each tuple $r \in D$ and the set of queried values $q$. Based thereon, the top- $k$ tuples may be identified in terms of their relevance to the given query (i.e., the set of queried values).

Let $R=\left(r_{1}, \ldots, r_{k}\right)$ be a relevance-ordered sequence of $k$-tuples, i.e., it holds that $r_{i} \in D$ and $i<j$ implies that $f^{*}\left(r_{i}, q\right) \leq f^{*}\left(r_{j}, q\right)$. The sequence is referred to as a top- $k$ result, denoted by $R^{*}=\left(r_{1}^{*}, \ldots, r_{k}^{*}\right)$, if for any other relevance-ordered sequence of $k$-tuples $R^{\prime}=\left(r_{1}^{\prime}, \ldots, r_{k}^{\prime}\right)$ it holds that $\sum_{i=1}^{k} f^{*}\left(r_{i}^{\prime}, q\right) \leq \sum_{i=1}^{k} f^{*}\left(r_{i}^{*}, q\right)$. We phrase the problem of identifying a top- $k$ result as follows:

Problem 1 (Multi-Modal Information Retrieval). Given a multi-modal query $\mathcal{Q}$ and a set of tuples $D$, the problem of multi-modal information retrieval is to retrieve a top- $k$ result, i.e., a relevance-ordered sequence of $k$-tuples $R^{*}=$ $\left(r_{1}^{*}, \ldots, r_{k}^{*}\right)$.

The relevance function $f^{*}$ is commonly unknown. Hence, any IR system will employ its own relevance function $f$ that aims to approximate $f^{*}$. The quality of the employed relevance assessment may then be quantified based on some ground truth information, i.e., a top- $k$ result for a specific multi-modal query. To this end, let $R=\left(r_{1}, \ldots, r_{k}\right)$ be a relevance-ordered sequence of $k$-tuples returned for a multi-modal query by an IR system that employs a relevance function $f$. Then, we assess the quality of the result retrieved through this relevance function by the normalised discounted cumulative gain [20]:

$$
n D C G(f)=\frac{\sum_{i=1}^{k} \frac{f\left(r_{i}, q\right)}{\log _{2}(i+1)}}{\sum_{i=1}^{k} \frac{f\left(r_{i}^{*}, q\right)}{\log _{2}(i+1)}} .
$$

Note that $n D C G(.) \in[0,1]$, while a value of 1.0 indicates perfect result quality.

\section{Approach OVERVIEW}

In this work, we propose an approach for multi-modal information retrieval that is generally applicable in diverse domains. In $\S 3.1$, we first explain the design principles for our approach. Then, in $\S 3.2$, we discuss several core concepts and representations, before $\S 3.3$ summarises our overall approach to multi-modal information retrieval.

\subsection{Design Principles}

A generic approach to multi-modal information retrieval shall respect the following principles:

(DP1) Domain-independence: An IR approach should unify the relevance computation of tuples regardless of the modalities that are considered in a particular domain. Traditional systems for uni-modal IR learn an embedding for one modality and define relevance based on a distance between the resulting embeddings [19]. However, the respective embedding model is not applicable for another modality and the choice of a distance function also depends on the application in question.

(DP2) Fusion-independence: State-of-the-art approaches for multi-modal IR treat each modality separately, which requires several passes over the data. Moreover, the final step of fusing the results of several uni-modal queries is challenging since the rankings obtained for different modalities are incomparable [4]. Multi-modal IR should, therefore, avoid any dependency implied by the need to fuse the results of different modalities.

(DP3) Embedding-independence: Vectorization of diverse modalities leads to different embedding spaces, so that additional reconciliation of vector-spaces is needed [21]. This, however, introduces a potential source of errors, so that multi-modal IR should not depend on such reconciliation. Moreover, the chosen embedding technique shall be invariant to certain transformations of heterogeneous data, such as rotation and illumination for images or watermarking and time-scale modification for audio and video data [16], [22], [23].

\subsection{Core Concepts and Representations}

Our approach to multi-modal IR is based on the notion of a heterogeneous information network, which we summarize below before turning to the definition of its schema and its vectorized representation.

Heterogeneous Information Networks (HIN). A HIN is a undirected graph $G=(V, E)$ with typed nodes $V$ and edges $E \subseteq[V]^{2}$ between them [24], where $[V]^{2}$ are all subsets of $V$ of size two. A type function $\phi: V \rightarrow \mathcal{A}$ maps each node to a modality $\phi(v)$. A node represents a value of its assigned type. Specifically, the value may be a vector of features of its modality (aka intrinsic characteristics of the node), such as the audio representation of a song.

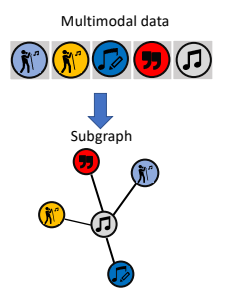

Fig. 2. A HIN.

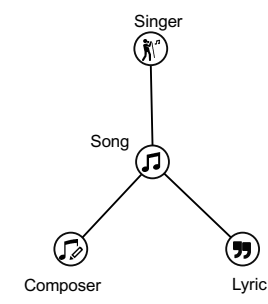

Fig. 3. A HIN schema.
Example 4. The construction of a HIN from a multi-modal tuple is illustrated in Fig. 2. Here, node types include a title, a singer, lyrics, and an audio representation of a song. The edges capture the relations between the nodes. For instance, an edge represents the fact that the respective singer has recorded the song. 
TABLE 1

Overview of important notations.

\begin{tabular}{ll}
\hline Notation & Explanation \\
\hline $\mathcal{D}=\left\{d_{1}, \ldots, d_{n}\right\}$ & A multi-modal database of tuples \\
$\mathcal{A}=\left\{A_{1}, \ldots, A_{m}\right\}$ & A set of modalities \\
$\mathcal{Q}=\left\{Q_{1}, \ldots, Q_{m}\right\}$ & A multi-modal query: A set of uni-modal queries \\
$\Omega=\cup_{A \in \mathcal{A}} A$ & All possible values of modalities \\
$q=\cup_{Q \in \mathcal{Q} Q}$ & The set of queried values \\
$G=(V, E)$ & A heterogeneous information network (HIN) \\
$\mathcal{G}=(\mathcal{V}, \mathcal{E})$ & A HIN schema \\
\hline
\end{tabular}

HIN schema. To capture the characteristics of the entities represented by nodes types in a HIN, a HIN schema specifies multi-modal constraints by restricting the types of nodes that can be connected by edges. A HIN schema is a graph $\mathcal{G}=(\mathcal{V}, \mathcal{E})$ where $\mathcal{V} \subseteq \mathcal{A}$ are modalities and $\mathcal{E} \subseteq[\mathcal{V}]^{2}$ are edges between them. A HIN $G=(V, E)$ is an instance of the HIN schema $\mathcal{G}$, if for all nodes node $v \in V$ it holds that $\phi(v) \in \mathcal{V}$ and for all edges $\left\{v_{1}, v_{2}\right\} \in E$ it holds that $\left\{\phi\left(v_{1}\right), \phi\left(v_{2}\right)\right\} \in \mathcal{E}$. The definition of a HIN schema is akin to the indexing of database attributes in IR frameworks [14].

Example 5. For our running example, a HIN schema as shown in Fig. 3. It comprises four modalities, singer, song, composer, and lyric. The previous HIN in Fig. 2 is an instance of this schema.

Network embedding. A vectorized representation of a HIN may be derived by network embedding, which is realized by an encoder and a decoder [25]. The former is a function $h_{\theta}: V \rightarrow \mathcal{R}^{d}$ that constructs a $d$-dimensional vector (where $d \ll|V|$ ), aka embedding, for the node. The latter is a function that maps these vectors to domain-specific quality metrics. In case of a graph-based model, the decoder is typically a similarity metric that reflects the proximity of two embeddings in the vector space. The accuracy of the representation of the proximity between nodes by the similarity of their embeddings is captured by a loss function, denoted by $L$. When learning an embedding model, one tries to minimize this loss function by finding an optimal set of parameters for the encoder and decoder.

Table 1 gives an overview of the most important notations used throughout the paper.

\subsection{Multi-Modal IR based on Network Embedding}

Using the above concepts and representations, the problem of multi-modal information retrieval as phrased in Problem 1 is lifted to HINs. That is, retrieval aims at the extraction of a top- $k$ result, but not in terms of tuples, but in terms of nodes of a target modality:

Problem 2 (HIN Information Retrieval). Given a multimodal query $\mathcal{Q}$ and a HIN $G$, the problem of HIN information retrieval with target modality $A$ is to retrieve a relevance-ordered sequence of $k$ nodes $R_{v}^{*}=\left(v_{1}^{*}, \ldots, v_{k}^{*}\right)$ with $\phi\left(v_{i}\right)=A$.

Once the respective nodes have been retrieved by solving Problem 2, the result for Problem 1 can be derived: For each retrieved node, a tuple is constructed by concatenating its value with those of neighbouring nodes in the HIN.

Following the idea to solve the problem of multi-modal IR through HIN IR, Fig. 4 gives an overview of our approach. In the first step, tuples are transformed into a

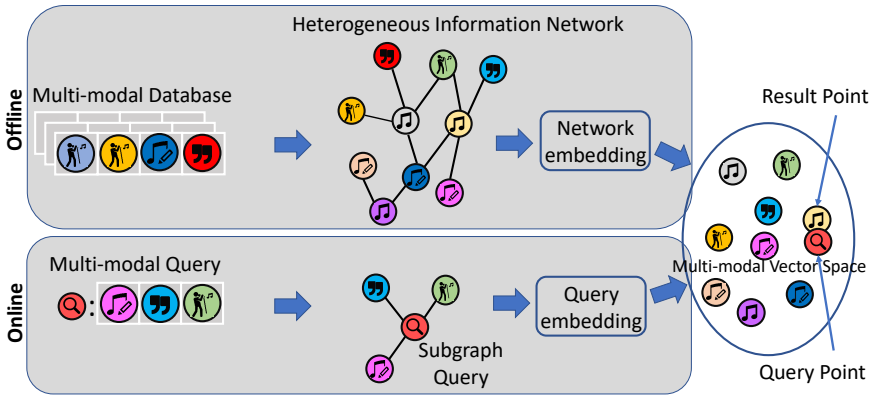

Fig. 4. The proposed approach to multi-modal information retrieval.

single HIN to represent their relations and modalities. Based thereon, a network embedding is constructed for each node of the HIN. This embedding captures both the intrinsic characteristics of the nodes as well as their relations in the HIN. This construction, detailed in $\S 4$, is done once and the results are indexed to speed up retrieval.

Given the queried values of a multi-modal query, we construct a query embedding in the same space. Such integration of different modalities at the query level enables query processing with a single pass over the data. The embedding of the queried values is explained in $\S 5$.

The retrieval of tuples that are relevant to the query is then grounded in the respective embeddings. Given the embedding of the query, $z_{q}$, and the set of embeddings of nodes of the HIN, $Z$, we identify the $k$ nodes of the HIN that have embeddings $z_{v} \in Z$ closest to $z_{q}$, according to some similarity function $s\left(z_{q}, z_{v}\right)$ (e.g., Euclidean similarity).

Turning to the design principles of $\S 3.1$, the universal nature of the HIN model enables us to achieve the required independence properties: Relevance computation becomes independent of the modalities and similarity measures employed in a specific domain (DP1). Moreover, our approach does not depend on fusion of retrieval results obtained for separate modalities (DP2). Finally, by constructing query embeddings in the embedding space of the data, we avoid the need for potentially erroneous reconciliation (DP3).

\section{Heterogeneous Network Embedding}

This section introduces our process of embedding a multimodal database. We first show how to construct a HIN from the database ( $₫ 4.1)$. Then, we propose a novel model for network embedding based on message-passing ( $\$ 4.2)$, which is subsequently instantiated to embed the HIN ( $\$ 4.3)$.

\subsection{HIN Construction}

Given a HIN schema, the construction of HIN for a multimodal database $D$ is done in a bottom-up manner. First, for each tuple $d \in D$, we construct a subgraph. The nodes are the respective attribute values of the tuple, i.e., $V_{d}=\left\{a_{i} \in d\right\}$, and while edges are defined between all pairs of values for which the HIN schema defines an edge between the respective attributes. Fig. 2 illustrates this construction. The subgraphs created for all tuples are then connected through their shared nodes (of the same type) to construct the final HIN. Fig. 5 gives an example where three subgraphs are connected through nodes that represent the same singer and the same composer. 


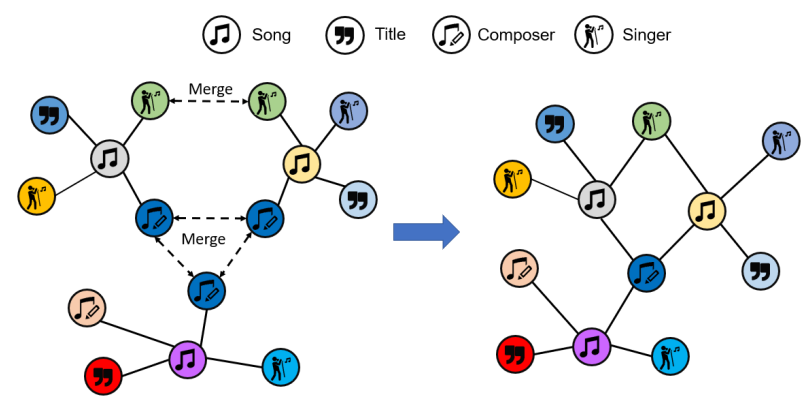

Fig. 5. A HIN for music information retrieval (right) is created by merging shared nodes of different subgraphs of multimodal data tuples (left).

Example 6. Consider two tuples representing songs: 'Paint it, Black' and 'Sympathy for the Devil'. To construct a HIN, we derive a subgraph for the first tuple with the respective song node; a singer node 'The Rolling Stones'; composer nodes 'Mick Jagger' and 'Keith Richards'; and a lyrics node. The nodes are connected according to the HIN schema in Fig. 3. Given the subgraph for the second tuple, the two subgraphs are connected since they share the compose nodes for 'Mick Jagger' and 'Keith Richards'.

\subsection{Network Embedding with Message-Passing}

Requirements. A model for network embedding that shall be applied for HINs should capture the following aspects:

1) Connectivity: Embeddings for nodes shall capture their relations. Following the homophily principle, the distance of nodes in the graph should be reflected by the distance of their embeddings in the embedding space.

2) Node types: The different types of nodes shall be incorporated in the embeddings.

3) Node values: The values of nodes, potentially involving multiple features that capture the nodes' characteristics, shall be represented in the embeddings.

A graph convolutional network (GCN). Given the above requirements, a Graph Convolutional Network (GCN) [26], [27] provides a starting point for an embedding model. A GCN directly reflects the connectivity of the original graph as well as the node values that are given as feature vectors. A traditional GCN applies a $k$-hop aggregation, which is a function that incorporates the values of the neighbourhood of a node through recursive computation with a predefined depth $k$. Here, the initial embedding of the recursion is given by the node value. If the value is undefined, the embedding is initialized randomly or through a one-hot vector of its degree [28].

Yet, a traditional GCN is agnostic to the types of nodes and $k$-hop aggregation would always incorporate the complete neighbourhood of a node. Applying such a model to a HIN would mix up the values of different modalities.

A GCN with message-passing. To overcome this limitation and achieve fine-granular control about the interplay of neighbouring nodes in the construction of an embedding, we propose a new GCN model based on messagepassing [29], [30]. Each forward pass then includes three phases, also illustrated in Fig. 6: (i) a sending phase, in which messages are broadcast to neighbours; (ii) a receiving phase, in which messages from neighbours are consumed; and (iii) a updating phase, which updates the embeddings.
Sending: Given a node $v$ at the $l$-th iteration, we send a message to its neighbours constructed from its current embedding $z_{v}^{(l)}$ :

$$
m_{v \rightarrow u}^{(l)}=M^{(l)} z_{v}^{(l)}
$$

where $M^{(l)}$ is a matrix that differs between iterations.

Receiving: For a node $v$, the messages received from its neighbours are aggregated into a community embedding:

$$
z_{N(v)}^{(l)}=\operatorname{agg}_{l}\left(\left\{m_{u \rightarrow v}^{(l)}, \forall u \in N(v)\right\}\right)
$$

where $a g g_{l}$ is an aggregation function and $N(v)$ is the set of neighbours of $v$. Informally, the community embedding $z_{N(v)}$ reflects how node $v$ is related to its neighbours.

Updating: For a node $v$, a new embedding $z_{v}^{(l+1)}$ is computed based on its current embedding $z_{v}^{(l)}$ and its community embedding $z_{N(v)}^{l}$ :

$$
z_{v}^{(l+1)}=\operatorname{combine}_{l}\left(z_{N(v)}^{(l)}, z_{v}^{(l)}\right)
$$

where combine $_{l}$ is a vector aggregation function that balances the intrinsic characteristics of a node and the influence of its neighbours.

Functions $a g g_{l}$ and combine $e_{l}$ are parametrized and in each iteration, we may use a different function. In a traditional GCN, the aggregation is commonly defined as a component-wise maximum of the neighbouring embeddings after applying some linear transformation [28]:

$\operatorname{agg}_{l}\left(\left\{m_{u}^{(l)}, \forall u \in N(v)\right\}\right)=\max \left(\left\{\sigma\left(W_{a g g}^{(l)} m_{u}^{(l)}+b^{(l)}\right), \forall u \in N(v)\right\}\right)$

Function combine $_{l}$ is typically a simple concatenation of the neighbourhood embedding $z_{N(v)}^{l}$ and the current embed$\left.\operatorname{ding} z_{v}^{(l)}\right)$, before applying some non-linear transformation:

$$
\operatorname{combine}_{l}\left(z_{N(v)}^{(l)}, z_{v}^{(l)}\right)=\sigma\left(W_{\text {concat }}^{(l)} \operatorname{concat}\left(z_{N(v)}^{(l)}, z_{v}^{(l)}\right)\right)
$$

In this setting, $M^{(l)}, W_{a g g}^{(l)}, b^{(l)}, W_{\text {concat }}^{(l)}$ are the parameters of the GCN that need to be learned.

\subsection{HIN Embedding}

The above GCN with message-passing mechanism enables us to incorporate types of nodes in the construction of embeddings, as required for HINs. To this end, we provide an instantiation of the above model, which we refer to as a heterogeneous GCN, or $h$-GCN for short.

$h$-GCN. The model adapts the message-passing procedure and incorporates node types (i.e., the modalities of the HIN) in the sending and receiving phases.

Sending: The sent message now depends on the type $s=$ $\phi(v)$ of the sending node $v$ and the type $t=\phi(u)$ of the receiving node $u$ :

$$
m_{v \rightarrow u}^{(l)}=M_{s, t}^{(l)} z_{v}^{(l)}
$$

where $M_{s, t}^{(l)}$ is a separate matrix for each pair of types $(s, t)$.

Receiving: Instead of using the maximum as an aggregation function, we sum up the messages from neighbours of a node $v$ to avoid the loss of modality information.

$$
a g g_{l}\left(\left\{m_{u \rightarrow v}^{(l)}, \forall u \in N(v)\right\}\right)=\sum_{u \in N(v)} \sigma\left(W_{a g g}^{(l)} m_{u \rightarrow v}^{(l)}+b^{(l)}\right)
$$




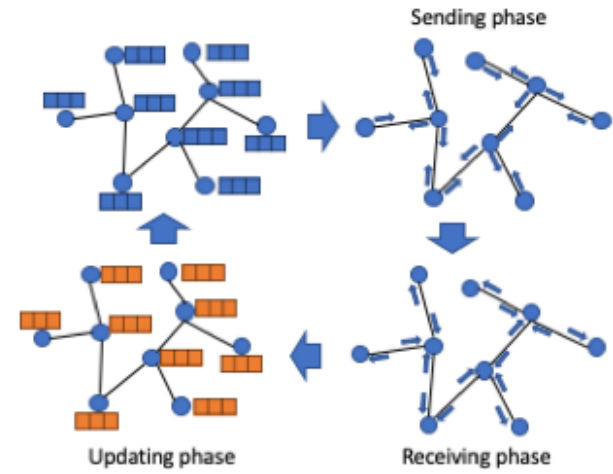

Fig. 6. GCN with message-passing.

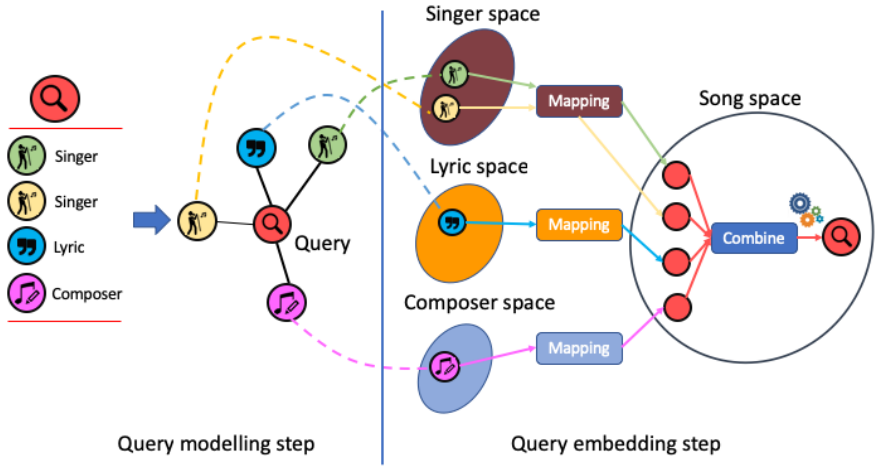

Fig. 7. From a multi-modal query to a graph model to a unified embedding.
The idea being that neighbouring nodes potentially belong to different modalities, so that the sum retains information about all of them. An aggregation based on the maximum, as in a traditional GCN, in turn, would only keep the information of one modality. Moreover, it is known that an aggregation based on the maximum, in some cases, cannot distinguish between two different neighbourhoods [31].

Algorithm. We describe the procedure to embed a HIN with a $h-\mathrm{GCN}$ in Alg. 1 . The algorithm corresponds to the forward pass in a deep learning setting, in which the parameters of the $h$-GCN $\left(M_{s, t}^{(l)}, W_{a g g}^{(l)}, b^{(l)}, W_{\text {concat }}^{(l)}\right)$ have already been derived. Initially, these parameters are assigned randomly, while, later, they are learned gradually using Stochastic Gradient Descent (SGD), taking into account a loss function.

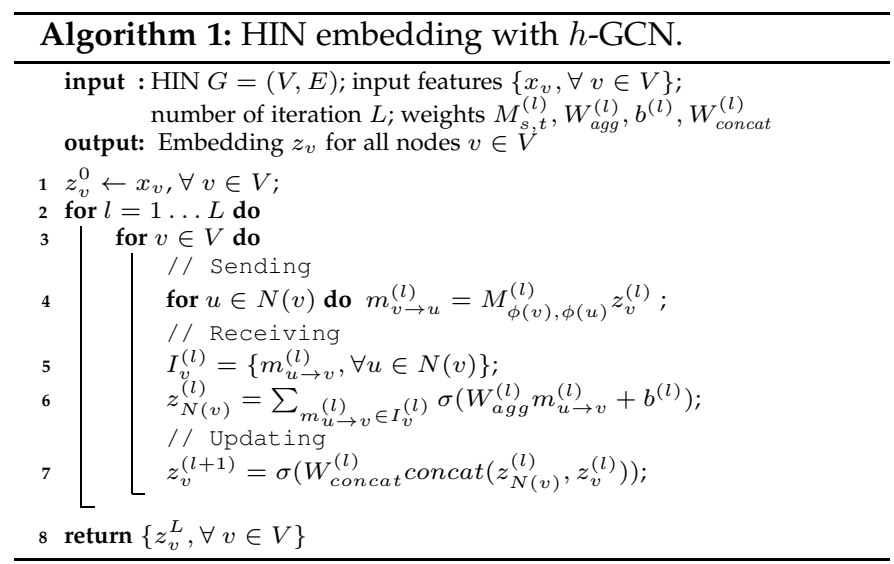

Alg. 1 starts by assigning an embedding to each node based on the node's features. Then, in each iteration $l$, the following steps are performed for each node: sending, receiving, and updating. For each node $v$ in the HIN, a message is sent to its neighbour $u$ (line 4). The message is constructed based on the node types $\phi(v)$ and $\phi(u)$. In the receiving step, given the messages that a node $v$ received from its neighbours $I_{v}^{(l)}$, the function $a g g_{l}$ is applied to obtain the community embedding of node $v$, i.e., $z_{N(v)}^{(l)}$ (lines 5-6). In the updating step, the new embedding of $v$ at iteration $l+1$ is obtained by applying the function combine $e_{l}$ to the community embedding $z_{N(v)}^{(l)}$ and its embedding of the previous iteration $z_{v}^{(l)}$. Finally, after $L$ iterations, we return the obtained embeddings (line 8).
Parameter Learning. We learn the model parameters, i.e., the message matrix $M$ and parameters of functions $a g g$ and combine, using SGD over the following loss function:

$$
L\left(z_{v}\right)=-\log \left(\sigma\left(z_{v}^{T} z_{u}\right)\right)-Q \cdot E_{u_{n} \sim P_{n}(v)} \log \left(\sigma\left(-z_{v}^{T} z_{u_{n}}\right)\right)
$$

This loss function minimizes (maximizes) the distance between embeddings of nodes that are close (distant) in the original HIN. Here, the exact definition of which nodes are deemed close and distant depends on a chosen notion of proximity (e.g., 1-hop or 2-hop). That is, $P_{n}(v)$ is a sampling function to select distant nodes (since their number is commonly much larger than the number of close nodes) and $Q$ is the number of samples.

\section{Embedding Multi-Modal Queries}

In this section, we show how to embed a multi-modal query, in the same space as the embedding of the HIN. Below, we first discuss how to transform a multi-modal query into a subgraph query ( $\$ 5.1)$, before turning to its embedding ( $\$ 5.2)$ and the question of how to learn the parameters for the embedding model ( $\$ 5.3)$.

\subsection{From Multi-modal Queries to Subgraph Queries}

Recall that a multi-modal query is represented by a set of queried values $q \subseteq \Omega$ (see $\{2.2$ ). Since a database is modelled using a $\operatorname{HIN} G=\{V, E\}$, a query $q$ corresponds to a subgraph of $G$ with a set of nodes $q \subset V$. Also, according to Problem 2, each query $q$ is associated with a modality (type) of interest $t \in \mathcal{A}$, denoted by a query subscript, $q_{t}$. Hence, the result for a query $q_{t}$ is a set of nodes of type $t$.

Example 7. Fig. 7 illustrates a query of a user looking for a song by specifying information on two singers, keywords in the lyrics, and a composer. In a first step, this query is converted into a subgraph.

\subsection{Multi-modal Query Embedding}

To facilitate retrieval, an embedding of a query $q$ needs to be in the same space as the embedding of the database, i.e., the HIN. In addition, the query embedding must be constructed based on all information available. Since $q$ is a set of queried values and each value is captured by a node $(q \subset V)$ for which an embedding is available already, the problem becomes the combination of embeddings of queried values to represent the whole query. 
A straight-forward approach to query embedding is to take the average over the respective embeddings of nodes. That is, the embedding of $q$, denoted by $z_{q}$, is computed as $z_{q}=(1 /|\{v \in q\}|) \sum_{v \in q} z_{v}$. By computing a linear combination of node embeddings, the resulting query embedding is in the same space as the HIN embedding. However, this approach neglects the multi-modal nature of the query, since the types of queried values are not distinguished. Below, we address this shortcoming.

Multi-modal space mapping. To incorporate the different types of queried values, we adopt the following view when constructing a query embedding: The nodes of each type are mapped into an embedding space that is specific to that type. For instance, we cannot expect the representation of a singer to be in the same space as the representation of a song title. However, the embeddings of a set of singers will be in the same space.

Adopting this view, we construct the embedding for a query $q_{t}$ by mapping the embeddings of the queried values (and, thus nodes) of all types to the embedding space of $t$, the type of interest. Subsequently, the mapped embeddings are combined to obtain the unified embedding for the whole query. To this end, we define two embedding operators, which, following [32], are referred to as mapping $P$ and combination $C$ of the embeddings of nodes in the query. While operator $P$ maps an embedding from one space for a type to another one, operator $C$ combines information from several embeddings in the space of a single type.

Mapping operator: The mapping operator $P$ takes as input an embedding $z_{v}$ of a node $v$ of type $s$ and a destination type $t$. It returns a new embedding $z_{v}^{\prime}$, i.e., $z_{v}^{\prime}=P\left(z_{v}, s, t\right)$. The new embedding $z_{v}^{\prime}$ can be considered as the embedding of $v$ in the space of type $t$. Formally, $P$ is defined as:

$$
z^{\prime}=M(z, s, t)=M_{s, t} z
$$

where $M_{s, t} \in R^{d \times d}$ is a mapping matrix from type $s$ to $t$. The matrix $M_{s, t}$ needs to be learned during a training phase.

Combination operator: This operator combines different embeddings in the same space. As there is no ordering of the queried values (i.e., the nodes) in query $q_{t}$, the operator shall be invariant to input permutation. For a set of embeddings $Z=z_{1}, \ldots, z_{m}$ of type $t$, it returns an embedding $z^{\prime}$ :

$$
z^{\prime}=C\left(\left\{z_{1}, \ldots, z_{m}\right\}\right)=M_{t} \sigma(\phi(Z))
$$

where $\sigma$ is a non-linear function, such as the Rectified Linear Unit (ReLU), $\phi$ is a permutation-invariant function that can be applied on set, and $M_{t}$ is a matrix that is trained for type $t$. In its simplest form, the operator is defined with $\phi$ being the summation and $M_{t}$ as the identity matrix:

$$
z^{\prime}=\sigma\left(\sum_{i} z_{i}\right)
$$

Algorithm. Given the operators $P$ and $C$, Alg. 2 captures the process of constructing an embedding for a query $q_{t}$.

The algorithm starts with the embedding of each node $v$ of query $q_{t}$. It converts the embedding of each node to the space of type $t$ using the mapping operator (line 3), which yields a set of mapped embeddings $Q$ (line 4). Finally, we apply the combination operator $C$ to the set $Q$ to obtain the query embedding $z_{q}$ (line 5).

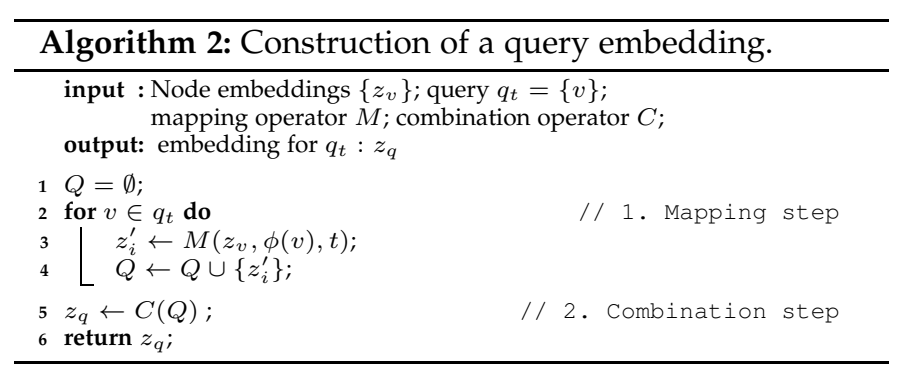

Example 8. Taking up Example 7, after obtaining the graph of the query, the mapping operator converts the singer, lyric, and composer embeddings to the embedding space of a song, see Fig. 7. Note that the respective embeddings of the singers, lyric, and composer are available from the construction of an embedding for the HIN. Then, the mapped embeddings are combined to obtain the query embedding.

A major advantage of our framework is its flexibility, as the training of the query embedding process is independent of the HIN embedding. Hence, we learn the parameters of operators $P$ and $C$ using the available HIN embedding.

\subsection{Parameter Learning}

To learn the parameters of the operators for the query embedding process, we need to define (i) a loss function that captures our objective, and (ii) training samples used to optimize the parameters. From Problem 1, we derive that training data shall contain pairs of a query and a top- $k$ result, $\left(q, R^{*}\right)$. This would allow to optimize the parameters to obtain a retrieval result $R$ for query $q$, such that $R$ approximates $R^{*}$. However, such training data is commonly not available and their manual construction is infeasible, as a large amount of queries would be required.

Generate sample queries. Against this background, we follow a training approach that employs top-1 queries. Such an approach has the advantage that training is efficient, since the result for each query is a single node. The generated sample queries are then used to define our loss function.

Given a type $t$, we define a training set of top- 1 queries that have as a result a single node of type $t$. We consider queries of varying levels of precision based on the number of neighbouring nodes of the result node that are part of the query. The most precise queries are defined as follows:

$$
\mathbb{Q}_{t}^{(1)}=\{(q, R)\}=\{(N(v),(v)), \forall v \in V: \phi(v)=t\} .
$$

That is, if the query contains all neighbours of a node $v, v$ is the best retrieval result. We generalize this idea to the set of queries with $\rho$-precision. It includes all queries that remove at most $\rho$ - 1 neighbours of a node $v$, when querying for $v$. The set of respective queries is defined recursively:

$$
\begin{gathered}
\mathbb{Q}_{t}^{\rho}=\mathbb{Q}_{t}^{(\rho-1)} \cup \\
\left\{\left(N^{\prime},(v)\right), \forall v \in V, N^{\prime} \subset N(v): \phi(v)=t \wedge\left|N(v) \backslash N^{\prime}\right|=\rho-1\right\} .
\end{gathered}
$$

Note that, with larger $\rho$, queries become less precise and may not have a unique answer any more. As such, relatively small values should be used for parameter $\rho$.

Loss function. In our setting of vector-based retrieval, a query embedding shall be close to the embeddings of 
the queries constructed above. That is, given the training queries, we expect $\operatorname{dist}\left(z_{q}, z_{v}\right)$ to be small where $q$ and $v$ is a pair of a training query and a node of the result of a top-1 query with $\rho$-precision, whereas the distance shall be large for all other nodes. We therefore define a loss function based on triplet loss [33] as follows:

$$
L\left(z_{q}, z^{+}, z^{-}\right)=\frac{1}{2} \max \left(0, m+\operatorname{dist}\left(z_{q}, z^{+}\right)-\operatorname{dist}\left(z_{q}, z^{-}\right)\right)
$$

where $z_{q}, z^{+}, z^{-}$are the embeddings of the query, a node of the query result (i.e., a positive sample), and node that is not part of the query result (i.e., a negative sample), respectively, and $m$ is a margin. This function is minimal, when $\operatorname{dist}\left(z_{q}, z^{-}\right)-\operatorname{dist}\left(z_{q}, z^{+}\right)>m$, i.e., when the distance between embeddings of the positive sample and the query is small, and the distance between the query embedding and the negative sample is large.

\section{Embedding LARge Networks}

Several directions may be followed to scale the construction of embeddings to large networks. Most prominently, model parallelism may be employed [34]. However, these approaches have the drawback that they require a strong coordination between the parallel processes used for training, making them not fault-tolerant [35].

In this section, we therefore propose a method for network embedding that parallelizes the learning of parameters. Our approach involves two steps. First, we partition the HIN into several overlapping subnetworks and embed each of them separately. Second, we reconcile the embeddings obtained for different subnetworks since they are defined over different embedding spaces.

Network partitioning. Before partitioning the HIN, we first select anchor nodes that are shared among different subnetworks and serve as the basis for later reconciliation. We propose to select nodes have a high degree, i.e., they are well-connected, as anchors. Intuitively, when constructing the network embedding, these nodes will affect the embeddings of many nodes in their neighbourhood. Once anchors are mapped to the same embedding space, their neighbours are likely to be in the same embedding space as well.

Once anchor nodes have been selected, we leverage traditional network partitioning techniques such as hMetis [36] to split the HIN, excluding anchor nodes, into subnetworks. Subsequently, the anchor nodes are added again to the obtained subnetworks.

Reconciliation of subnetwork embeddings. To reconcile the embeddings obtained per subnetwork, we exploit the anchor nodes as illustrated in Fig. 8. The anchor nodes will be assigned different embeddings in different subnetworks, so that they indicate how the respective embedding spaces relate to one another.

Consider a partitioning of a given HIN into subnetworks $H_{1}, \ldots, H_{k}$, for which we obtained the embeddings $Z_{1}, \ldots, Z_{k}$ for the nodes of the respective subnetworks. We strive for a mapping of embeddings $Z_{1}, Z_{2}, \ldots, Z_{k}$ into a single embedding space, which, without loss of generality, is one of the $k$ spaces, denoted by $Z_{0}$. We propose to learn a mapping function $h\left(Z_{i}\right)$ (e.g., $h\left(Z_{i}\right)=W \times Z_{i}$ ) that takes a source set of embeddings as input and returns a mapped

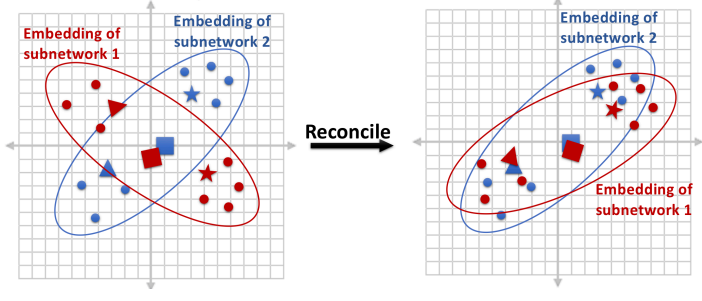

Fig. 8. Reconciliation of embeddings.

set of embeddings, such that the embeddings of the anchor nodes are close in $Z_{0}$. Our objective can be captured by the following loss function:

$$
L\left(h, Z_{i}, Z_{0}, T\right)=\sum_{v \in T}\left\|h\left(z_{i, v}\right)-z_{0, v}\right\|_{F}
$$

where $\|.\|_{F}$ is the Frobenius norm, $T$ is the set of anchor nodes, and $z_{i, v}$ is the embedding of anchor node $v$ in the embedding space of $Z_{i}$. The parameters of the mapping function $h\left(Z_{i}\right)$ are trained using SGD to minimize the above loss function. While the learning is guided solely by the anchor nodes, the resulting mapping function is able to map the whole embedding space, i.e., it is applicable to the embeddings of all nodes of the respective subnetwork.

\section{EXPERIMENTAL Results}

This section reports on an experimental evaluation of the proposed framework using a diverse set of real-world and synthetic datasets. Our experimental setup is discussed in (§7.1), before the following aspects are evaluated:

- The general efficiency of our approach ( $\$ 7.2)$.

- The effectiveness of HIN embedding ( $(77.3)$.

- The effectiveness of query embedding ( $\$ 7.4)$.

- The efficiency and effectiveness of information retrieval in comparison to baseline techniques ( $\$ 7.5)$.

- The scalability through network partitioning (§7.6).

\subsection{Setup}

Datasets. We use four real-world datasets from a wide range of domains, see Table 2.

TABLE 2

Statistics for real-world datasets.

\begin{tabular}{lllll}
\hline Dataset & flickr & dbis & fma & citation \\
\hline \#Nodes & $19^{\prime} 471$ & $150^{\prime} 858$ & $244^{\prime} 360$ & $1^{\prime} 511^{\prime} 035$ \\
\#Edges & $105^{\prime} 425$ & $708^{\prime} 502$ & $426^{\prime} 296$ & $2^{\prime} 084^{\prime} 019$ \\
\hline
\end{tabular}

Real-world datasets: The flickr dataset includes several modalities, including images, image groups, terms, and users [24]. Relations link images and users, images and terms, and images and groups. The dbis dataset [37] includes modalities such as papers, authors, venues, and terms in the research domain of databases and information systems. Relations link papers and authors, papers and venues, and papers and terms. The fma dataset [38] contains songs and related information such as short audio segments, albums, artists, and genres. We enrich the fma dataset with album arts, further increasing its heterogeneity. Relations link songs with the other types, except album arts, which are linked to albums. 
For each of these datasets, we generate 100 multi-modal queries of varying sizes, ranging from 1 to 10 . The values for a query are selected using truncated random walks around a node in the respective HIN. This way, we ensure that the queries are semantically meaningful. For each set of queries of the same size, we ensure that the query contains different modalities (1-3 for flickr/dbis and 2-4 for fma). The retrieved result is judged by three hired experts, who score the relevance of top- 20 results on a scale of 0 to 5 .

For a scalability analysis, we use the citation dataset [39] with more than $1 \mathrm{M}$ nodes. It contains different modalities, including authors, affiliations, bibliographic metrics, and research interests. This dataset is intended to evaluate the efficiency of our approach to network embedding that employs a partitioning scheme, as proposed in $\S 6$.

Synthetic datasets: In addition to the real-world datasets, we also rely on synthetic data for sensitivity analysis. In particular, we study the effects of dataset size and the number of modalities, which includes several control parameters: $m$, the number of tuples; $\alpha$, the HIN density (\#tuples/\#nodes); $n$, the number of nodes; $H$, the structure of the HIN schema; $l$, the heterogeneity degree (\#attributes); and $\delta$, the distribution controlling how many attributes are HIN nodes. We first generate $n$ nodes and then divide the nodes into $l$ clusters according to $\delta$. Then, we construct $m$ tuples by selecting from each attribute a set of nodes according to a HIN schema. Given the tuples and the schema $H$, we can construct the HIN following the process described in $\S 4$. If not stated otherwise, we use the following parameter configurations: $n=1 M, m=500 K, l=5, \delta$ is a uniform distribution for $l-1$ attributes while the last attribute contains $m$ nodes, and $H$ is a star schema.

Evaluation Metrics. Two main metrics are used:

Runtime: We evaluate efficiency in terms of the retrieval time needed to answer a multi-modal query, and the training time required to construct embeddings.

Normalized Discounted Cumulative Gain (nDCG): The effectiveness is evaluated using $\mathrm{NDCG}$ (see also $\S 2.3$ ), a stateof-the-art IR metric that incorporates the relevance and ranking position of results.

Hyperparameters. Unless stated otherwise, we use the parameters suggested in [28] for network embedding. The learning rate is set to 0.0001 for all experiments. The batch size for experiments with real-world data is 256, and 16 for the synthetic data. As for the loss function (Eq. 4), for each node, we use 50 random walks with a length of 5 . All models use a Rectified Linear Unit as a non-linear function.

Regarding the GCN, we found that by training the node embeddings at iteration 0 together with the model, we achieve better results. Following [28], for the aggregation and combine functions, we use $\max$ and concat, respectively. Regarding the query embedding, the margin for the loss function in Eq. 7 is set to 0.1, as suggested in [33].

Environment. All experiments have been obtained on an Intel Core i7-6700K server with a NVIDIA GTX 1080Ti and $32 \mathrm{~GB}$ RAM. For k-NN search, we leverage $\mathrm{nmslib}^{1}$ for indexing, which facilitates fast retrieval.

${ }^{1}$ https:/ / github.com/nmslib/

\subsection{General Efficiency}

We analyse the general efficiency of our approach in terms of its runtime, using the synthetic dataset.

Model for query embedding. We assess the training time needed to learn the parameters for query embedding (see $\S 5.3)$, relative to the HIN's density level and its number of nodes. Fig. 9 depicts the runtime (in minutes) for different configurations.
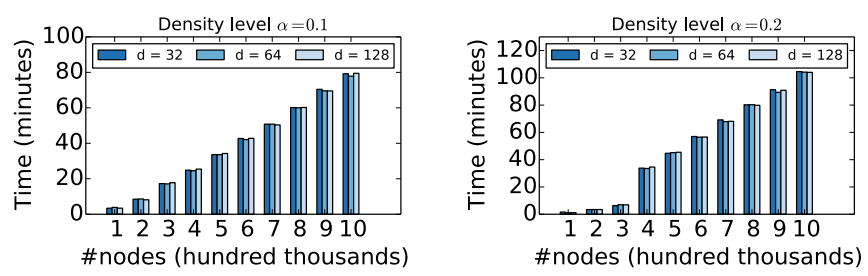

Fig. 9. Training time for the model for query embedding vs. \#nodes.

In general, the training time increases linearly with the number of nodes in the HIN. This is expected as the number of training queries increases linearly w.r.t the number of nodes. For example, the training time is $3 \mathrm{~min}$ when the network size is $100 \mathrm{k}$, but increases to $79 \mathrm{~min}$ when the network size is $1 \mathrm{M}$. On the other hand, the embedding size has little effect on the training time.
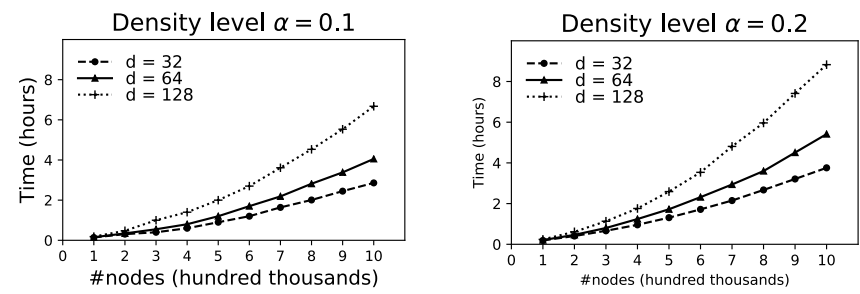

Fig. 10. Training time of the whole framework vs. \#node.

End-to-end embedding co-training. We further analyse the training time of the end-to-end process, incorporating both models for query and network embedding, see Fig. 10. The measured training time is higher than solely for the model for query embedding (Fig. 9), due to a higher overall number of parameters. Yet, it is significantly lower than the sum of training times if the models for query and network embedding are learned separately. For instance, for a network with 500k nodes, the end-to-end process for co-training takes around $2 \mathrm{~h}$, whereas isolated training would require $3 \mathrm{~h}$ for network embedding and $0.5 \mathrm{~h}$ for query embedding.

Retrieval time. Finally, we evaluate the time required to answer a query using our proposed approach, relative to the query size and the number of nodes in the HIN. Fig. 11 illustrates that, unlike training times, retrieval times are extremely short. For instance, for a query of size nine and a network with $1 \mathrm{M}$ nodes, retrieval is done in $23 \mathrm{~ms}$. Also, the query size has little effect on the retrieval time. Also, for common queries of rather small size, there is virtually no difference in retrieval time. For example, increasing the query size from three to nine, for a network of 200k nodes, the retrieval time remains stable at $4 \mathrm{~ms}$.

A key observation is that the embedding size does not affect the retrieval time. The is explained by the fact that we need to consider solely the relative difference between the 
embeddings. By indexing these differences beforehand, we remove any effect of the embedding size on retrieval time. Another observation is that the retrieval time scales linearly with the network size. This is expected as increasing the network size also increases the retrieval space.
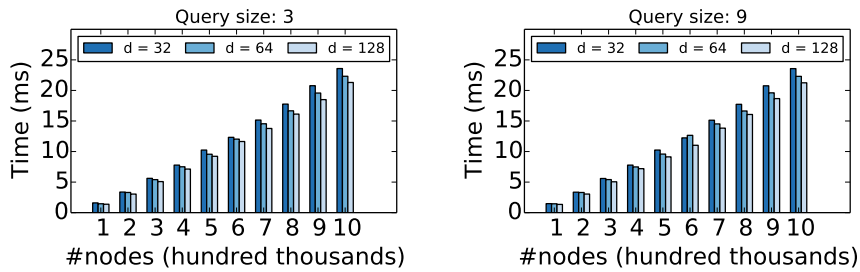

Fig. 11. Effects of query size on retrieval time.

\subsection{Effectiveness of HIN Embedding}

Quantitative evaluation. This experiment compares our embedding model, $h$-GCN as presented in $\S 4.3$, against two baselines, i.e., traditional GCN [28] and node2vec, in terms of the normalized Discounted Cumulative Gain (nDCG). The embedding of a query is generated by averaging the embeddings of all nodes in the query. Moreover, we employ the loss function defined in Eq. 4.
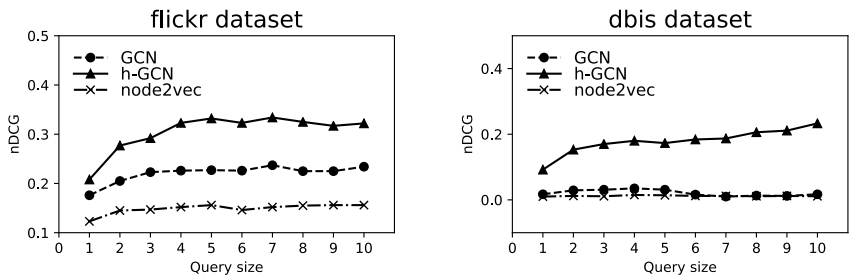

Fig. 12. Effectiveness of HIN embedding.

Fig. 12 shows the nDCG (the higher, the better) related to the query size. Clearly, our model outperforms all baselines by a large margin. For example, using the dbis dataset, $h$ GCN achieves an nDCG of 0.184 , which is $11.5 \times$ better than the best baseline, when the query size is five. The worst results are obtained with node2vec, since it neglects intrinsic characteristics of nodes.

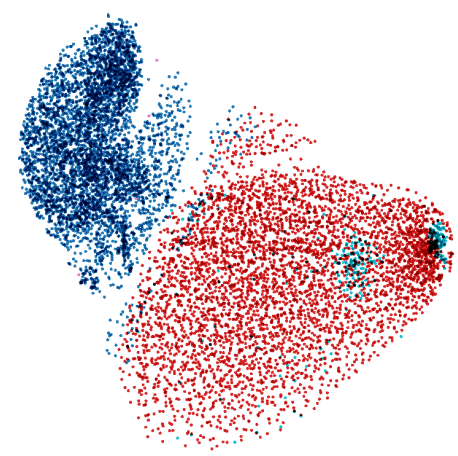

Fig. 13. Visualization of the embeddings.

Qualitative evaluation. Fig. 13 shows the t-SNE visualization of node embeddings for the flickr dataset. It illustrates three clusters: terms (red), images (blue) and users (light blue). This confirms our hypothesis that the terms, images, users belong to different embedding spaces.

\subsection{Effectiveness of Query Embedding}

To evaluate the effectiveness of our approach to query embedding, we first construct the network embedding for the HIN using traditional GCN. Based on the node embeddings, we then learn the query embedding following Alg. 2. Our model is compared with a baseline which computes the query embedding by averaging the query nodes. The comparison is performed with different query sizes.
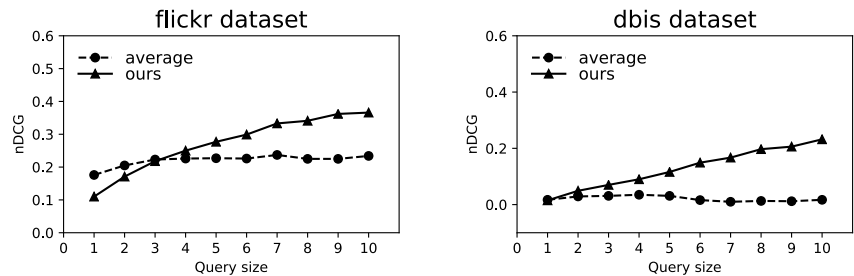

Fig. 14. Comparison of different query embedding methods.

Fig. 14 illustrates that the approach proposed in this paper outperforms the baseline technique. For the flickr dataset, the nDCG values are 0.3 and 0.22 , respectively, when the query size is six. Our approach achieves this improvement by incorporating the heterogeneous information available in the query to retrieve more relevant results.

\subsection{End-to-end Comparison}

Comparison with traditional baselines. We compare our approach with several traditional retrieval methods, such as tfidf [40], CCA [3], MSAE [1]. To make tfidf work in a multi-modal setting, we represent other modalities by their respective textual descriptions. For CCA and MSAE, which are cross-modal retrieval methods, we compare the obtained results with our method by performing retrieval from text to image on the flickr dataset and text to audio on the fma dataset. For the dbis dataset, CCA is ignored as it does not support text-to-text retrieval. We further vary the query heterogeneity, i.e., the number of considered modalities.

TABLE 3

Comparison with baselines in terms of $\mathrm{nDCG}$.

\begin{tabular}{lccc|ccc|ccc}
\hline Dataset & \multicolumn{3}{c}{ flickr } & \multicolumn{3}{c}{ dbis } & \multicolumn{3}{c}{ fma } \\
\hline $\begin{array}{l}\text { Query } \\
\text { hetero. }\end{array}$ & 1 & 2 & 3 & 1 & 2 & 3 & 2 & 3 & 4 \\
\hline tfidf & 0.36 & 0.37 & 0.35 & $\mathbf{0 . 4 7}$ & 0.45 & 0.49 & 0.42 & 0.47 & 0.52 \\
cca & 0.42 & 0.45 & 0.44 & N/A & N/A & N/A & 0.52 & 0.54 & 0.57 \\
msae & 0.52 & 0.51 & 0.55 & 0.36 & 0.37 & 0.33 & 0.57 & 0.62 & N/A \\
Ours & $\mathbf{0 . 5 5}$ & $\mathbf{0 . 6 3}$ & $\mathbf{0 . 8 7}$ & 0.41 & $\mathbf{0 . 7 7}$ & $\mathbf{0 . 9 3}$ & $\mathbf{0 . 8 3}$ & $\mathbf{0 . 8 4}$ & $\mathbf{0 . 8 7}$ \\
\hline
\end{tabular}

Table 3 shows that our method generally outperforms the baseline techniques. It achieves an nDCG value of 0.63 , whereas CCA reaches 0.45, MSAE reaches 0.31, and tf-idf reaches 0.37 on the flickr dataset with a query heterogeneity of two. Our technique yields better results than CCA and MSAE, since these techniques handle image/audio/text as separate modalities. Both of them try to map different modalities to a pair of embeddings that are close. Yet, the embeddings for different modalities are learned separately, so that relations between them are not directly captured.

Also, as the query becomes more heterogeneous, the differences between our approach and the baseline techniques becomes larger. This is due to the baselines not leveraging the query heterogeneity in their models. 
Moreover, tfidf yields the best results for the dbis dataset with a query heterogeneity of one, since tfidf is optimized for text-to-text retrieval. Yet, our method performs better than tfidf when the query becomes more heterogeneous.

Comparison with multi-pass retrieval. Next, we compare our approach that requires only a single pass over the data with baseline techniques that combine the results from several passes over the data, one per queried modality. Specifically, we use the tfidf baseline to retrieve results using each attribute separately and then combine their rankings. We also construct another baseline based on tfidf, which computes the representation for each attribute separately and generates the query embedding by averaging the attribute representations. The two baselines represent early and late fusion approaches in heterogeneous retrieval, respectively.

The effectiveness and efficiency of the considered approaches is visualised in Fig. 15. Here, our technique clearly dominates in terms of retrieval quality. While all techniques have a similar retrieval time for a query heterogeneity of one, our technique is able to retrieve answers faster than fusion-based approaches for heterogeneous queries. This trend stems from late fusion requiring several passes over the data, while early fusion requires several passes over the query to construct the embedding.

\subsection{Scalability through Network Partitioning}

Finally, we evaluate the efficiency of our approach to embedding through network partitioning, as introduced in $\S 6$. Measuring the end-to-end training time, we explore the influence of the \#nodes and \#subnetworks. We keep the density level $\alpha=0.1$ and the embedding size at 32. Instead of training all subnetworks in a parallel manner, we train the subnetworks in a sequential manner and report the average training time for all subnetworks.

In Fig. 16, increasing the network size, the training time increases. However, this trend is much less pronounced when using multiple subnetworks. For instance, with four subnetworks, the training time is only $30 \mathrm{~min}$, instead of $2 \mathrm{~h}$ in the sequential case (network with $1 \mathrm{M}$ nodes). We also evaluate the scalability of our approach when applied to the citation dataset using five subnetworks. We observe that the training time is extremely short, around $40 \mathrm{~min}$, for an embedding size of 32 . This shows that our method is able to handle large networks.

Concerning the effectiveness, we observe a slight decrease in the quality of retrieval results when increasing the number of subnetworks. That is, without network partitioning, we obtain an nDCG value of 0.874 for the flickr dataset. With four subnetworks, this value is reduced to 0.827 . The reason for this effect are neglected connections between the subnetworks. However, the reduction in effectiveness is relatively moderate.

\section{Related Work}

Heterogeneous information network. The notion of a HIN was first proposed in [24], for the setting of similarity search. Since then, HINs have been used in various applications [13], [14], such as clustering [41], [42], link prediction [43], or recommendation [44]. In these applications, a
HIN enables to capture complex relations between data tuples, so that meta-paths are used to define tuple similarity, as required for classification, clustering, and recommendation.

Our approach is the first to apply a HIN in an information retrieval setting [14]. Unlike the aforementioned techniques that are based on an assessment of meta-paths, we follow the paradigm of representation learning. That is, we represent the nodes of a HIN as embeddings in order to facilitate retrieval. The universal nature of our HIN model enables us to apply it for diverse retrieval settings that involve multiple modalities.

Multi-modal information retrieval. Multi-modal IR systems can process homogeneous queries or heterogeneous queries. While systems to support homogeneous queries are easier to implement and have been employed in firstgeneration commercial applications [2], [1], heterogeneous queries become more popular due to extending information needs of users. Yet, systems designed for homogeneous queries cannot directly handle heterogeneous data. It was therefore suggested to create a common representation of heterogeneous data [2], [45], [3], before transforming a homogeneous query to this common space and determining the relevant results via nearest-neighbour search.

To provide more direct support for querying heterogeneous data, cross-modal retrieval systems, such as [3], [6], have been proposed. Here, a query is specified in one modality, while the retrieved results are captured in another modality. Most of the existing systems focus on two modalities, though: images and texts. Such models cannot be extended for more than two modalities easily.

Moreover, systems that support queries with multiple modalities have been proposed [46], [3], [6]. These systems answer a multi-modal query through a combination of several uni-modal queries. Some systems perform late fusion [7], [8], [9] by solving querying each modality separately and then merge the respective results. For instance, in [7], results for images and texts are obtained separately and then combined. Due to the need to pass over the data multiple times, these systems show performance issues. Other systems employ early fusion [10], [11], [12], which computes different representations for the modalities and embeds the multi-modal queries into these vector spaces. While this avoids multiple passes over the data, the representation ignores the relations between modalities, thereby breaking the semantic structure of the data. As a consequence, features of retrieved elements may not belong to the same data entity. Going beyond the state-of-the-art, we process multi-modal queries with a single pass over the data, while incorporating the relations between modalities.

Network embedding. Representation learning for a network constructs a vector model of the nodes, such that the node representations reflect the network structure [47], [25]. Approaches for network embedding are distinguished based on how the network structure is incorporated. Shallow encoders [48], [49] compute for each node a unique embedding directly. They are typically fast to train and can be used without initial node features. Deep encoders [28] compute a node embedding based on its neighbourhood.

While our network embedding can be seen as a deep encoder, it differs from traditional approaches as we also 

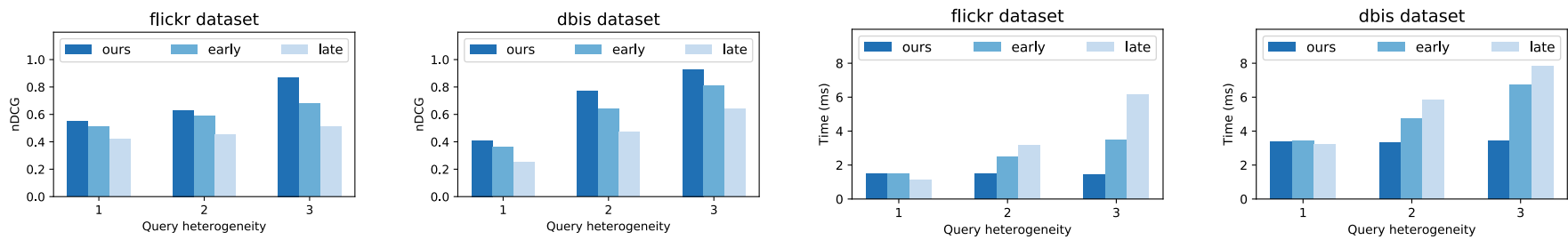

Fig. 15. Our technique for one pass retrieval vs techniques for multi-pass retrieval.
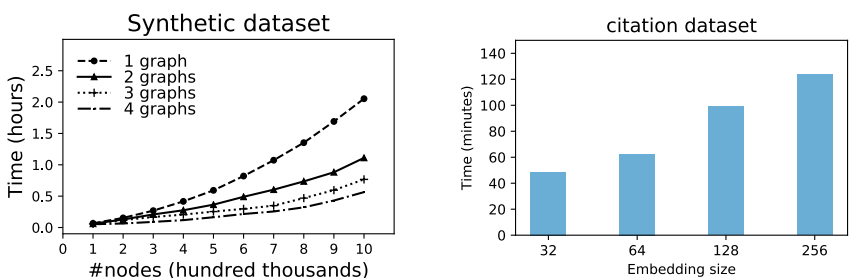

Fig. 16. The effect of network partitioning on the training time.

capture the heterogeneity of the nodes in the embeddings. Closest to our work is the HIN embedding proposed in [50]. However, this approach is limited in various aspects: It does not support multiple queries, neglects the transformation from multi-modal data to a HIN, and does not scale to large networks. The approach presented in this article overcomes these limitations.

Turning to the specifics of representing the network structure, most approaches are based on a random walk to capture a node's neighbourhood [48], [49]. Two nodes are considered to be close, if one occurs on the random walk from the other one. Network embedding based on random walks can be considered as an application of word embeddings for graphs. Other approaches to capture the network structure consider two nodes to be similar, if their neighbourhoods are highly overlapping [51], [52], which is commonly referred to as second-order node similarity. Yet, all these approaches target a different use case compared to the one addressed here: We do not aim to preserve the network structure, but strive for a network embedding that is suitable for multi-modal information retrieval.

\section{Conclusions}

In this paper, we presented a new direction for multimodal IR that relies on an embedding of a heterogeneous information network. Such a HIN enables us to capture the rich semantics of both, multi-modal data and queries. Based thereon, we proposed a novel network embedding model to obtain a vectorized representation of a HIN and a technique to construct a query embedding based on the HIN embedding through mapping and combination operators. As a result, we obtain embeddings that are defined over the same space to achieve accurate relevance assessment. We further introduced a network partitioning scheme to increase the scalability of the network embedding. Our experimental results for real-world and synthetic datasets illustrate the efficiency and effectiveness of our approach.

In future work, we will explore the dynamic evolution of a network to enable the retrieval of data that has not been considered in the training phase. This way, we aim at support for applications based on streaming data.

\section{REFERENCES}

[1] W. Wang, B. C. Ooi, X. Yang, D. Zhang, and Y. Zhuang, "Effective multi-modal retrieval based on stacked auto-encoders," in $V L D B$, 2014, pp. 649-660.

[2] W. Wang, X. Yang, B. C. Ooi, D. Zhang, and Y. Zhuang, "Effective deep learning-based multi-modal retrieval," VLDBJ, 2016.

[3] M. Dorfer, J. Schlüter, A. Vall, F. Korzeniowski, and G. Widmer, "End-to-end cross-modality retrieval with cca projections and pairwise ranking loss," IJMIR, vol. 7, no. 2, pp. 117-128, 2018.

[4] S. Sattari and A. Yazici, "Multimodal query-level fusion for efficient multimedia information retrieval," IJIS, pp. 2019-2037, 2018.

[5] D. Vandic, S. Aanen, F. Frasincar, and U. Kaymak, "Dynamic facet ordering for faceted product search engines," TKDE, vol. 29, no. 5 , pp. 1004-1016, 2017.

[6] Y. Cao, M. Long, J. Wang, and S. Liu, "Collective deep quantization for efficient cross-modal retrieval," in AAAI, 2017, pp. 3974-3980.

[7] H. J. Escalante, C. A. Hérnadez, L. E. Sucar, and M. Montes, "Late fusion of heterogeneous methods for multimedia image retrieval," in ICMR, 2008, pp. 172-179.

[8] A. Mourão, F. Martins, and J. Magalhães, "Multimodal medical information retrieval with unsupervised rank fusion," CMIG, vol. 39, pp. 35-45, 2015.

[9] B. Safadi, M. Sahuguet, and B. Huet, "When textual and visual information join forces for multimedia retrieval," in ICMR, 2014.

[10] H. Xu, K. He, L. Sigal, S. Sclaroff, and K. Saenko, "Textto-clip video retrieval with early fusion and re-captioning," arXiv:1804.05113, 2018.

[11] P. Budikova, M. Batko, and P. Zezula, "Fusion strategies for largescale multi-modal image retrieval," in TLDKS, 2017, pp. 146-184.

[12] S. Sattari and A. Yazici, "Multimedia information retrieval using fuzzy cluster-based model learning," in FUZZ-IEEE, 2017, pp. 1-6.

[13] Y. Sun and J. Han, "Mining heterogeneous information networks: a structural analysis approach," KDD Expl. News., pp. 20-28, 2013.

[14] C. Shi, Y. Li, J. Zhang, Y. Sun, and S. Y. Philip, "A survey of heterogeneous information network analysis," TKDE, vol. 29, no. 1, pp. 17-37, 2016.

[15] L. Jin, L. Feng, G. Liu, and C. Wang, "Personal web revisitation by context and content keywords with relevance feedback," TKDE, vol. 29, no. 7, pp. 1508-1521, 2017.

[16] N. Abbadeni, "Information retrieval from visual databases using multiple representations and multiple queries," in SAC, 2009.

[17] L. Meng, A.-H. Tan, and D. Xu, "Semi-supervised heterogeneous fusion for multimedia data co-clustering," TKDE, 2013.

[18] L. Luo, D. Guo, X. Zhao, J. Wu, O. Rottenstreich, and X. Luo, "Near-accurate multiset reconciliation," TKDE, pp. 952-964, 2018.

[19] P. Jing, Y. Su, L. Nie, X. Bai, J. Liu, and M. Wang, "Low-rank multiview embedding learning for micro-video popularity prediction," TKDE, vol. 30, no. 8, pp. 1519-1532, 2017.

[20] K. Järvelin and J. Kekäläinen, "Cumulated gain-based evaluation of ir techniques," TOIS, vol. 20, no. 4, pp. 422-446, 2002.

[21] Z. Wang, Q. Lv, X. Lan, and Y. Zhang, "Cross-lingual knowledge graph alignment via graph convolutional networks," in EMNLP, 2018, pp. 349-357.

[22] N. Abbadeni, "An approach based on multiple representations and multiple queries for invariant image retrieval," in VISUAL, 2007, pp. 570-579.

[23] M. Long, J. Wang, J. Sun, and S. Y. Philip, "Domain invariant transfer kernel learning," TKDE, vol. 27, no. 6, pp. 1519-1532, 2014.

[24] Y. Sun, J. Han, X. Yan, P. S. Yu, and T. Wu, "Pathsim: Meta path-based top-k similarity search in heterogeneous information networks," in VLDB, 2011, pp. 992-1003.

[25] W. L. Hamilton, R. Ying, and J. Leskovec, "Representation learning on graphs: Methods and applications," DEBU, 2017. 
[26] M. Defferrard, X. Bresson, and P. Vandergheynst, "Convolutional neural networks on graphs with fast localized spectral filtering," in NIPS, 2016, pp. 3844-3852.

[27] T. N. Kipf and M. Welling, "Semi-supervised classification with graph convolutional networks," ICLR, 2017.

[28] W. Hamilton, Z. Ying, and J. Leskovec, "Inductive representation learning on large graphs," in NIPS, 2017, pp. 1024-1034.

[29] J. Gilmer, S. S. Schoenholz, P. F. Riley, O. Vinyals, and G. E. Dahl, "Neural message passing for quantum chemistry," in ICML, 2017.

[30] K. Aberer, P. Cudré-Mauroux, and M. Hauswirth, "A framework for semantic gossiping," SIGMOD, vol. 31, no. 4, pp. 48-53, 2002.

[31] K. Xu, W. Hu, J. Leskovec, and S. Jegelka, "How powerful are graph neural networks?" arXiv preprint arXiv:1810.00826, 2018.

[32] W. Hamilton, P. Bajaj, M. Zitnik, D. Jurafsky, and J. Leskovec, "Embedding logical queries on knowledge graphs," in NIPS, 2018.

[33] F. Schroff, D. Kalenichenko, and J. Philbin, "Facenet: A unified embedding for face recognition and clustering," in CVPR, 2015.

[34] J. Geng, D. Li, and S. Wang, "Rima: an rdma-accelerated modelparallelized solution to large-scale matrix factorization," in ICDE, 2019, pp. 100-111.

[35] C. T. Duong, H. Yin, T. D. Hoang, T. G. L. Ba, M. Weidlich, Q. V. H. Nguyen, and K. Aberer, "Parallel computation of graph embeddings," arXiv preprint arXiv:1909.02977, 2019.

[36] G. Karypis and V. Kumar, "Metis-unstructured graph partitioning and sparse matrix ordering system, version 2.0," 1995.

[37] Y. Dong, N. V. Chawla, and A. Swami, "metapath2vec: Scalable representation learning for heterogeneous networks," in $K D D$, 2017, pp. 135-144.

[38] M. Defferrard, K. Benzi, P. Vandergheynst, and X. Bresson, "Fma: A dataset for music analysis," in ISMIR, 2017, pp. 1-8.

[39] J. Tang, J. Zhang, L. Yao, J. Li, L. Zhang, and Z. Su, "Arnetminer: extraction and mining of academic social networks," in KDD, 2008.

[40] J. Leskovec, A. Rajaraman, and J. D. Ullman, Mining of massive datasets. Cambridge university press, 2014.

[41] Y. Sun, Y. Yu, and J. Han, "Ranking-based clustering of heterogeneous information networks with star network schema," in KDD, 2009, pp. 797-806.

[42] C. Luo, W. Pang, and Z. Wang, "Semi-supervised clustering on heterogeneous information networks," in PAKDD, 2014.

[43] B. Cao, X. Kong, and S. Y. Philip, "Collective prediction of multiple types of links in heterogeneous information networks," in ICDM, 2014, pp. 50-59.

[44] C. Shi, C. Zhou, X. Kong, P. S. Yu, G. Liu, and B. Wang, "Heterecom: a semantic-based recommendation system in heterogeneous networks," in KDD, 2012, pp. 1552-1555.

[45] Y. Cao, M. Long, J. Wang, Q. Yang, and P. S. Yu, "Deep visualsemantic hashing for cross-modal retrieval," in KDD, 2016.

[46] A. Yazici, M. Koyuncu, T. Yilmaz, S. Sattari, M. Sert, and E. Gulen, "An intelligent multimedia information system for multimodal content extraction and querying," MTA, pp. 2225-2260, 2018.

[47] H. Cai, V. W. Zheng, and K. Chang, "A comprehensive survey of graph embedding: problems, techniques and applications," TKDE, 2018.

[48] B. Perozzi, R. Al-Rfou, and S. Skiena, "Deepwalk: Online learning of social representations," in KDD, 2014, pp. 701-710.

[49] A. Grover and J. Leskovec, "node2vec: Scalable feature learning for networks," in KDD, 2016, pp. 855-864.

[50] C. T. Duong, H. Yin, D. Hoang, M. H. Nguyen, M. Weidlich, Q. V. H. Nguyen, and K. Aberer, "Graph embeddings for one-pass processing heterogeneous queries," in ICDE, 2020, pp. 1-4.

[51] S. Cao, W. Lu, and Q. Xu, "Grarep: Learning graph representations with global structural information," in CIKM, 2015, pp. 891-900.

[52] M. Ou, P. Cui, J. Pei, Z. Zhang, and W. Zhu, "Asymmetric transitivity preserving graph embedding," in KDD, 2016, pp. 1105-1114.

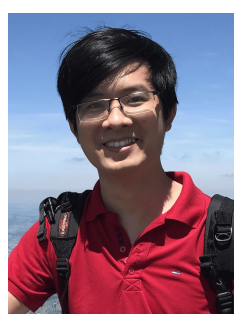

Duong Chi Thang is a Phd student at Ecole Polytechnique Federale de Lausanne (EPFL), Switzerland. He received the Master and Bachelor degrees in computer science from EPFL and from Ho Chi Minh University of Technology, Vietnam. His research interests include graph analysis, network embedding, network alignment. $\mathrm{He}$ has publications in significant journals and conferences such as VLDBJ, SIGMOD, and SIGIR.

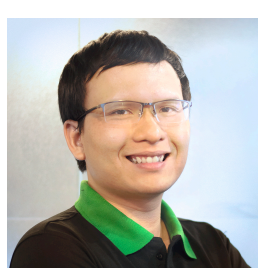

Nguyen Thanh Tam received the PhD degree in Data Science from EPFL, Switzerland. He holds a Master degree in Computer Science also from EPFL. His research interests include Data Filtering, Crowdsourcing, and Deep Learning for data lakes, data networks, and data streams. His research has been published in leading conferences and top-tier journals, such as VLDB, IJCAI, SIGIR, TKDE, and VLDBJ.

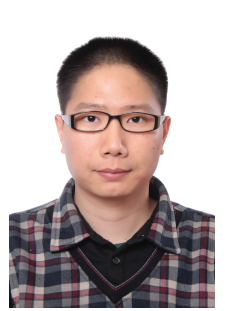

Hongzhi Yin received the $\mathrm{PhD}$ degree in computer science from Peking University, in 2014. He is a senior lecturer with the University of Queensland. He received the Australia Research Council Discovery Early-Career Researcher Award, in 2015. His research interests include recommendation system, user profiling,topic models, deeplearning, social media mining, and location-based services.

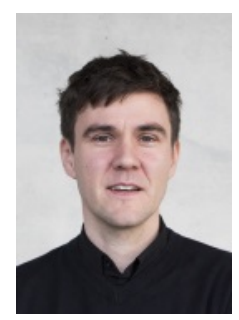

Matthias Weidlich is professor and Chair of Databases and Information Systems at the Department of Computer Science at HumboldtUniversität zu Berlin. His research focuses on process-oriented and event-based information systems, and his results appear regularly in premier conferences (SIGMOD, VLDB, ICDE) and journals (TKDE, Inf. Sys., VLDBJ) in the field. He serves as EiC for the Information Systems journal and on the ACM DEBS Steering Committee.

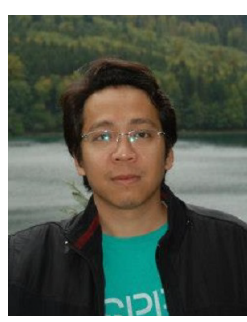

Mai Thai Son is an assistant professor at Queen's University Belfast, UK. Before that, he was doing research at University of Grenoble Alpes in France, Aarhus University in Denmark and Ludwig-Maximilians-University of $\mathrm{Mu}$ nich (LMU) in Germany. His research focuses on Machine Learning and Data Mining algorithms for large complex data.

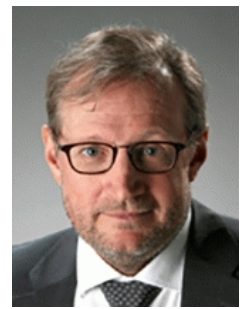

Karl Aberer is a full professor for Distributed Information Systems at EPFL, Switzerland. His research interests are on semantics in distributed information systems. He received his Ph.D. in mathematics in 1991 from the ETH Zurich. From 2012 to 2016 he was Vice-President of EPFL responsible for information systems. $\mathrm{He}$ is cofounder of LinkAlong, a startup for document analytics. He is member of the editorial boards of VLDB Journal, ACM TAAS and WWW Journal and chairman of the ICDE Steering Committee.

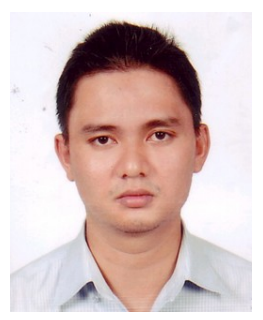

Nguyen Quoc Viet Hung is a Senior Lecturer in Griffith University. He earned his Master and PhD degrees from EPFL (Switzerland). He received the Australia Research Council Discovery Early-Career Researcher Award, in 2020. His research focuses on Data Integration, Data Quality, Information Retrieval, Trust Management, Recommender Systems, Machine Learning and Big Data Visualization, with special emphasis on web data, social data and sensor data. 